\title{
Workshop Papers and Authors
}

\author{
Presiding: Jeffrey A. Anderson
}

Lipid Peroxidation and Plant Tissue Disorders: Introduction to the Workshop

Jeffrey A. Anderson

Biological Roles and Biochemistry of the Lipoxygenase Pathway

Harold W. Gardner

Peroxidative Activity of Apple Peel in Relation to Development of Poststorage Disorders

Zhanyuan Du and William J. Bramlage

Release of Fluorescent Peroxidized Lipids from Membranes in Senescing Tissue by Blebbing of LipidProtein Particles

Katalin Hudak, Kening Yao, and J.E. Thompson

Toward a Comprehensive Model for Lipid Peroxidation in Plant Tissue Disorders

Robert L. Shewfelt and Albert C. Purvis

\section{Lipid Peroxidation and Plant Tissue Disorders: Introduction to the Workshop}

\author{
Jeffrey A. Anderson \\ Department of Horticulture and Landscape Architecture, Oklahoma State University, Stillwater, OK 74078
}

\begin{abstract}
Among the lipid peroxidation reactions, the addition of molecular $\mathrm{O}_{2}$ to polyunsaturated fatty acids (PUFAs) is of particular interest in biological systems. The lipoxygenase pathway is the most-studied enzymatic pathway for oxidizing PUFAs, but nonenzymatic reactions have also been characterized. When one considers the significance of lipid peroxidation, it is surprising to discover the many diverse areas that are affected. In fact, whether one considers the process beneficial or detrimental depends on the circumstances. In situations where lipid peroxidation has been implicated in tissue damage, controversy exists over whether it is involved in the primary events causing injury or merely a by-product.

Lipid peroxidation is involved with normal developmental processes, including production of flavor and odor volatiles, formation of compounds with growth-regulator-like activities, and senescence. Characteristic flavors and aromas, such as those associated with cucumber (Cucumis sativus L.) and tomato (Lycopersicon esculentum Mill.), are due in part to the presence of various enzymes in the respective lipoxygenase pathways (Gardner, 1989). Although desirable characteristics may be imparted to foods by oxidized lipids, rancidity and other off-flavors can also develop (Perkins, 1989).

Induced defenses, including the hypersensitive response associated with resistance to pathogens and herbivore defense involving proteinase inhibitors, rely in part on oxidation of PUFAs (Farmer and Ryan, 1992). These authors proposed that methyl jasmonate, formed from linolenic acid, may serve as a secondary messenger in the lipidbased signaling pathway. A direct role of aldehydes formed from PUFAs in inhibiting fungal growth also has been established (HamiltonKemp et al., 1992).
\end{abstract}

Approved for publication by the Director, Oklahoma Agricultural Experiment Station. The cost of publishing this paper was defrayed in part by the payment of page charges. Under postal regulations, this paper therefore must be hereby marked advertisement solely to indicate this fact.
Lipid peroxidation via free-radical-mediated reactions has been implicated in abiotic stresses. Evolution of ethane, a breakdown product of linolenic acid, was detected after temperature stresses (Harber and Fuchigami, 1986; Nanaiah and Anderson, 1992) and exposure to gaseous pollutants (Kimmerer and Kozlowski, 1982). Extensive peroxidation of membrane lipids may impair function and structure, possibly through the formation of gel-phase domains in the membrane (Pauls and Thompson, 1980). A similar decrease in membrane fluidity observed in senescing tissues was blocked by a lipoxygenase inhibitor (Fobel et al., 1987), but factors such as sterol content also may be important in determining membrane lipid viscosity (Duxbury et al., 1991).

Plant cells combat the formation of free radicals with antioxidants and free-radical scavengers. Leaf senescence mediated by free radicals was related to a decrease in superoxide dismutase and catalase activity (Dhindsa et al., 1981) —enzymes that detoxify superoxide and hydrogen peroxide, respectively. Purvis and Shewfelt (1993) proposed that an accumulation of stress-triggered oxidizing agents may overcome the defensive capabilities of the cell, leading to injury.

Lipid peroxidative reactions play key roles in plant responses to biotic and abiotic stresses, developmental processes, and food quality. The papers from this workshop cover many of these areas, beginning with an overview of the lipoxygenase pathway, focusing on the biochemistry and biological roles of the two branches of the lipoxygenase pathway. Presentations dealing with specific disorders are followed by a comprehensive model addressing the question of cause and effect.

\section{Literature Cited}

Dhindsa, R.S., P. Plumb-Dhindsa, and T.A. Thorpe. 1981. Leaf senescence: Correlated with increased levels of membrane permeability and lipid peroxidation, and decreased levels of superoxide dismutase and catalase. J. Expt. Bot. 32:93-101. 
Duxbury, C.L., R.L. Legge, G. Paliyath, R.F. Barber, and J.E. Thompson. 1991. Alterations in membrane protein conformation in response to senescencerelated changes. Phytochemistry 30:63-68.

Farmer, E.E. and C.A. Ryan. 1992. Octadecanoid precursors of jasmonic acid activate the synthesis of wound-inducible proteinase inhibitors. Plant Cell 4:129-134.

Fobel, M., D.V. Lynch, and J.E. Thompson. 1987. Membrane deterioration in senescing carnation flowers. Plant Physiol. 85:204-211.

Gardner, H.W. 1989. How the lipoxygenase pathway affects the organoleptic properties of fresh fruit and vegetables, p. 98-112. In: D.B. Min and T.H. Smouse (eds.). Flavor chemistry of lipid foods. Amer. Oil Chem. Soc., Champaign, Ill.

Hamilton-Kemp, T.R., C.T. McCracken, Jr., J.H. Loughrin, R.A. Andersen, and D.F. Hildebrand. 1992. Effects of some natural volatile compounds on the pathogenic fungi Alternaria alternata and Botrytis cinerea. J. Chem. Ecol. 18:1083-1091.

Harber, R.M. and L.H. Fuchigami. 1986. The relationship of ethylene and ethane production to tissue damage in frozen rhododendron leaf disks. J. Amer. Soc. Hort. Sci. 111:434-436.

Kimmerer, T.W. and T.T. Kozlowski. 1982. Ethylene, ethane, acetaldehyde, and ethanol production by plants under stress. Plant Physiol. 69:840-847.

Nanaiah, G.K. and J.A. Anderson. 1992. Electrolyte leakage and evolution of ethylene and ethane from pepper leaf disks following temperature stress and fatty acid infiltration. J. Amer. Soc. Hort. Sci. 117:846-851.

Pauls, K.P. and J.E. Thompson. 1980. In vitro simulation of senescence-related membrane damage by ozone-induced lipid peroxidation. Nature (London) 283:504-506.

Perkins, E.G. 1989. Gas chromatography and gas chromatography-mass spectrometry of odor/flavor components in lipid foods, p. 35-56. In: D.B Min and T.H. Smouse (eds.). Flavor chemistry of lipid foods. Amer. Oil Chem. Soc., Champaign, Ill.

Purvis, A.C. and R.L. Shewfelt. 1993. Does the alternative pathway ameliorate chilling injury in sensitive plant tissues? Physiol. Plant. 88:712-718.

\title{
Biological Roles and Biochemistry of the Lipoxygenase Pathway
}

\author{
Harold W. Gardner \\ National Center for Agricultural Utilization Research, Agricultural Research Service, U.S. Department of \\ Agriculture, Phytoproducts Research, 1815 North University Street, Peoria, IL 61604
}

Comparative overview of the plant and animal kingdoms. This workshop emphasized the "dark side" of uncontrolled lipid oxidation in plants, but another aspect of this oxidative process is thought to be a "normal" part of the physiological status of the plant and animal kingdoms. That is, the so-called lipoxygenase pathway is common to plants and animals (Fig. 1). Because the pathway in plants is activated by wounding and pathogen attack, the argument can be made that the pathway is normally quiescent. In animals, certain levels of prostaglandins and lipoxygenase products are known to be maintained, but only a few studies have addressed the endogenous levels of lipoxgenase metabolites in plants (e.g., see Vick and Zimmerman, 1982). More data are available concerning endogenous levels of the lipoxygenase pathway-generated jasmonic acid family of metabolites (see review in Hamberg and Gardner, 1992). Although some lipoxygenase isoenzymes can oxidize certain glyceride lipids, it is generally acknowledged that free polyunsaturated fatty acids are the preferred substrates. In plants, a little-known portion of the pathway is how the initial step is triggered, namely the lipolytic action on glycerides. Lipoxygenase oxidation of polyunsaturated fatty acids, and metabolism of the resulting fatty acid hydroperoxides, are more thoroughly characterized. However, the biosynthesis of prostaglandins via prostaglandin endoperoxide synthase has not yet been observed in plants. There are a few reports of prostaglandins isolated from plants, but few higher plants contain the arachidonic acid necessary for the action of prostaglandin endoperoxide synthase. Since prostaglandin-like fatty acids are formed simply by peroxidative reactions of hydroperoxides of either arachidonic acid or linolenic acid (O'Connor et al., 1984), it is unlikely that the "prostaglandins" found in plants resulted from true enzymic reactions of prostaglandin biosynthesis. Because higher plants largely metabolize their endogenous polyunsaturated fatty acids, linoleic and linolenic acids, and animals use mostly arachidonic acid in these reactions, they have been called the octadecanoid and eicosanoid pathways, respectively.

André and Hou first detected lipoxygenase in plants in 1932, but its significance was not understood until about 50 years later. Novel possible physiological roles for the lipoxygenase pathway in plants are now emerging rapidly. However, prostaglandin endoperoxide synthase

The cost of publishing this paper was defrayed in part by the payment of page charges. Under postal regulations, this paper therefore must be hereby marked advertisement solely to indicate this fact. and lipoxygenase activities in animals were discovered much later (Hamberg and Samuelsson, 1974; Nugteren et al., 1966), and their physiological roles were defined quickly thereafter. Although plants and animals diverged in their evolution more than 2.5 billion years ago, there remain several common enzymic reactions in the pathway in addition to lipoxygenase. Allene oxide synthase (formerly called hydroperoxide isomerase, hydroperoxide cyclase, and hydroperoxide dehydrase) is found in plants and coral (Fig. 1), and hydroperoxide lyase has been identified in plants and fish (see review in Gardner, 1991). Interestingly, both the octadecanoid and eicosanoid pathways are often triggered by stress. Many of the physiological responses are amelioration or repair of the effects of stress. For many years, aspirin has been known to block prostaglandin formation by inhibiting prostaglandin endoperoxide synthase activity. Recently, Peña-Cortés et al. (1993) reported that aspirin in plants also inhibits allene oxide synthase, the enzyme that produces the plant equivalent of prostaglandins (12oxo-phytodienoic acid).

An overview of the lipoxygenase or octadecanoid pathway of plants is shown in Fig. 2. After lipolytic action, the first enzyme in the series is lipoxygenase. Most plant lipoxygenase isoenzymes hydroperoxidize polyunsaturated fatty acids in a stereo-specific manner giving either $13(S)$ - or $9(S)$-hydroperoxides, and some yield a mixture of both. Only a few isoenzymes give a more racemic mixture, similar to fatty acid autoxidation products. Thus, the hydroperoxides usually obtained from linoleic acid are 13(S)-hydroperoxy-cis-9, trans11-octadecadienoic acid (13S-HPOD) and 9(S)-hydroperoxy-trans10, cis-12-octadecadienoic acid (9S-HPOD), and those obtained from linolenic acid are 13(S)-hydroperoxy-cis-9,trans-11,cis-15octadecatrienoic acid (13S-HPOT) and $9(S)$-hydroperoxy-trans-10, cis12,cis-15-octadecatrienoic acid (9S-HPOT). By Fischer convention the 13(S)- and $9(S)$-hydroperoxides are $(L)$ and $(D)$, respectively. After lipoxygenase action, there are several hydroperoxide-metabolizing branches of the pathway that impact the physiology of plants; these aspects are discussed in the sections below.

The jasmonic acid family. The key enzyme involved in the biosynthesis of the family of 7-iso-jasmonic acid, jasmonic acid, their esters, and numerous related metabolites was first observed in flaxseed (Linum usitatissimum L.) to transform 13S-HPOD into 12-oxo-13hydroxy-cis-9-octadecenoic acid ( $\alpha$-ketol).Zimmerman (1966) named this enzyme hydroperoxide isomerase. Subsequently, hydroperoxide isomerase was found in the germ of corn seed (Zea mays L.), and, 